\title{
SOCIEDAD DE RIESGO Y GESTIÓN. ESTUDIO DE CASO EN ORGANIZACIONES DEDICADAS AL COMERCIO DE ALIMENTOS
}

\author{
Caila, María de las Mercedes \\ Ulla Irigoyen, Verónica Liliana \\ Leda Ayelén Cecilia \\ Pérez Sebastián
}

\section{Resumen:}

El planeta afronta una pandemia en el marco de una coyuntura difícil que en algunas latitudes ha profundizado una crisis en términos socioeconómicos. Esta situación ha puesto a individuos y estados nacionales en escenarios adversos, donde la complejidad del problema dificulta las respuestas eficientes. Los actores sociales y productivos, deben enfrentar contextos de transformación que incluyen realidades concretas y formas de interpretarlas. La gestión cultural es un apoyo ante cambios tecnológicos.

Palabras clave: Sociedad de Riesgo; Complejidad, Gobernanza, Gestión Empresarial

\section{Abstract:}

The planet is currently facing a pandemic that in some parts of the world has worsened the difficult socioeconomic crisis they were undergoing. This desequilibrium has not helped people or nations to find efficient solutions. Social and productive actors, must face transformation in a context that includes concrete reality and its own ways of interpreting them. Cultural management is support in crisis o technological changes.

Key words: Risk Society, Complexity, Governance, Business Management.

\section{INTRODUCCIÓN}

Uno de los efectos de la pandemia, es que ha puesto en relieve las desigualdades existentes en las actividades productivas, principalmente en los desequilibrios regionales y las diferencias en la capacidad de acumulación. Todos estos factores son preexistentes a la misma, pero se han visto agravados por las restricciones que a nivel global se aplican para el resguardo de las condiciones sanitarias.

El PNUD indicó que en 2019 el $80 \%$ de la población mundial dispone de menos de 5\% de la riqueza. De acuerdo a OXFAM, el 1\% más rico de la población mundial posee 
más que el 99\% restante de las personas del planeta. ${ }^{1}$ Desde la CEPAL se estima que la región latinoamericana sufrirá este año una caída del $9,1 \%$ en el PIB, un aumento de $5,4 \%$ en la tasa de desocupación y un incremento del $7,1 \%$ de la pobreza en comparación con el mismo período del año anterior.

Como correlato se supone la desaparición de 2,7 millones de empresas, circunstancia que representa aproximadamente el 19\% de la región. ${ }^{2}$ De acuerdo con el Banco Mundial, las PYMES conforman aproximadamente el $90 \%$ del total de las empresas a nivel mundial, generan el $60-70 \%$ del empleo. Esto las convierte en importante actores económicos, y a su vez tienen menores condiciones para enfrentar satisfactoriamente turbulencias de magnitud importantes como la presente.

Antes del Covit 19, a nivel mundial, ya existían fuertes reclamos sociales en varios países y regiones; si bien contaban con distinta composición social y diferentes objetivos, todos realizan cuestionamientos a la excesiva concentración económica y a la desigual redistribución de beneficios. De modo que si bien las condiciones previas, o la hoy anhelada normalidad, no eran una panacea para las organizaciones productivas, el transcurso de las restricciones impuestas por la pandemia agravan y dificultan la misma subsistencia, en particular de aquellas pymes o emprendimientos que cuentan con menos herramientas en capital o en gestión.

\section{LA SOCIEDAD DE RIESGO}

La alteración de la vida cotidiana y productiva pone a la mayoría de los sujetos ante situaciones inéditas que deben enfrentar con pocos recursos. Sobre la situación actual el historiador Yuval Harari rememora una antigua y sutil maldición china que a su criterio se aplica al presente y que dice: "Ojalá vivas tiempos interesantes." Si la pandemia constituye "esos tiempos interesantes" a los que alude Harari; en tiempos anteriores también existían cuestionamientos a las estructuras sociales y económicas. De acuerdo a explicaciones dentro del campo de las ciencias sociales, nos encontramos en la llamada sociedad de riesgo, definida así por varios autores, entre ellos Beck, Giddens y Lash. "Expresándolo llanamente la individualización actual significa la desintegración de las certezas de la sociedad industrial y la compulsión de encontrar nuevas certezas para uno mismo y para quienes carecen de ellas. Pero

\footnotetext{
${ }^{1}$ De acuerdo al Informe del PNUD, los países de A. L. y C se encuentran entre los más desiguales del mundo en ingreso per cápita de los hogares: 10 de los 15 países más desiguales del mundo pertenecen a esta región (UNU/WIDER, 2008). El coeficiente de Gini del ingreso de A.L. y C es $65 \%$ más alto que en los países de ingresos altos, $36 \%$ más elevado que en los países del Este Asiático y $18 \%$ superior al del África Subsahariana

2 Para observar una contracción similar hace falta retroceder hasta la Gran Depresión de 1930

(-5\%), o hasta 1914 (-4,9\%). Datos : https://www.cepal.org/es/comunicados/pandemia-covid-19
} 
también significa nuevas interdependencias, incluso interdependencias globales" (Beck: 1997: 29)

Esta definición de sociedad de riesgo se diferencia de las sociedades modernizadas en la industrialización tradicional, en la explicación de la cohesión social y funcionamiento institucional. En éstas últimas, la categoría de ciudadano lograba la conformación de una identidad regulada por instituciones adecuadas a requerimientos del individuo, hecho que fortalece la cohesión social. Dentro de este esquema tradicional, en su vida cotidiana los sujetos enfrentan constantemente circunstancias cuyo impacto o riesgo se tornan relativos por la inclusión como ciudadano y por las certezas del funcionamiento institucional.

Pero desde hace un tiempo relativo, vivimos en la denominada sociedad de riesgo, en la cual pueden presentarse problemáticas divergentes de los objetivos de las instituciones. Esto significa que existe una inadecuación en los antiguos soportes culturales de las sociedades industriales, quedando el individuo con la percepción de aislamiento respecto de su entorno institucional, es decir en una sociedad de riesgo. En la sociedad de riesgo lo imprevisible conduce a la incertidumbre, porque las medidas organizativas y los procedimientos de decisión no son pertinentes a los problemas del individuo y en consecuencia la cohesión social se debilita.

Siguiendo a Beck (2002:22-29), en las últimas décadas se han privilegiado tecnologías y sistemas que conducen a la contaminación, erosión de los suelos, deforestación, recalentamiento global, pérdida de diversidad biológica, etc. No ha sido obstáculo para continuar con este proceso devastador la repetición de catástrofes que muestran la falacia de los mecanismos de gestión del riesgo. Todo lo cual no hace más que reafirmar la prevalencia de los intereses económicos por sobre los sociales. Las decisiones políticas fundamentales -traducidas en acciones o abstenciones- se toman priorizando los requerimientos del mercado, al margen de los intereses colectivos. De hecho se han presenciado advertencias sobre el surgimiento de pandemias importantes, existieron casos que se pudieron controlar, sin que esto haya impactado en ninguna disposición para mejorar las condiciones en que se realiza la producción (en particular de alimentos, aun cuando todas están interrelacionadas). ${ }^{3}$

Vimos que las sociedades se cohesionan y se desarrollan en aspectos, económicos, productivos y culturales, a partir de la creencia en que sus instituciones sociales generan estabilidad y contención relativa entre individuos, debiendo cuidar y prevenir todo aquello que pueda significar un problema a la vida y salud de los miembros. La

\footnotetext{
${ }^{3}$ En este contexto -apunta Beck- los peligros se minimizan mediante cálculos que llegan a resultados que sólo son riesgos, se limitan por medio de comparaciones y se normalizan jurídica y científicamente como "riesgos residuales e improbables", de manera que se estigmatizan las protestas como brotes de "irracionalidad"
} 
pandemia actual posiblemente ponga en cuestión estas certezas sobre la racionalidad de nuestros sistemas, algo típico de la sociedad de riesgo. Si este planteo es objetivo o subjetivo, no resulta algo prioritario ya que igualmente esto quedará como percepción de la realidad en los sujetos, orientando modificaciones en todo su accionar.

Este impacto afecta tanto a la política como a la organización económica, movilizando la búsqueda de estrategias y previsiones posibles ante un escenario todavía no definido totalmente y pleno de incertidumbres.

\section{CASOS ANALIZADOS}

A partir del análisis de algunas pautas que conforman parte de la cultura, en el caso de dos emprendimientos de la ciudad de Rosario entrevistados, se observarán algunos ejes que cueste modificar, y prácticas de la gestión sostenidas durante esta coyuntura. Comprendemos la cultura organizacional como un repertorio principalmente de hábitos, prácticas y valores, que fortalecen la cohesión organizacional y contribuyen al logro de sus objetivos. La pandemia transmuta las relaciones en el trabajo y fuera de él. Ha transformado la forma de organización del tiempo, de las tareas y fundamentalmente altera prioridades en los objetivos organizacionales y las formas conocidas de lograrlos. Por lo tanto caracterizaremos algunas pautas centrales de las dos organizaciones conforme a las características señaladas.

El caso de un Mercado Popular (MP) que inicia sus actividades en 2015, en un espacio compartido, luego de consolidar a un grupo de productores en una feria itinerante que funcionaba desde inicios de esa década ${ }^{4}$.

Con un régimen de propiedad cooperativo de hecho ${ }^{5}$, este mercado comercializa productos de primera necesidad (principalmente alimentación e higiene) de productores de la economía social y popular. Se busca: activar cadenas cortas de comercialización que minimicen la figura de los intermediarios, practicar el comercio justo que asegure precios accesibles al consumidor y viabilizar la actividad del productor a través de una percepción de ingresos sostenible.

El nacimiento y consolidación del MP significa para quienes allí comercializan y trabajan una experiencia colectiva para hacer frente a los riesgos sociales y económicos de una sociedad que se muestra macroeconómicamente endeble.

\footnotetext{
${ }^{4}$ Según nos comentaban en entrevista dicha feria presentaba puntos fijos en la ciudad que iban rotando acompañando el itinerario del camión del Programa Nacional "Pescados para Todos", de la Secretaría de Comercio Interior 2009-2015.

${ }^{5}$ Sus integrantes se encontraban a principios del presente año aun en tratativas para formalizar la cooperativa.
} 
Se constituye en punto neurálgico de una red de actores colectivos e individuales de la economía social y popular. Las vinculaciones con productores/as, incluso, implica su incorporación en procesos decisorios. Si bien, las decisiones cotidianas son tomadas por encargados/as del MP, las de comercialización e incorporación de productos nuevos son discutidas en reuniones con quienes abastecen al MP y tienen particular interés en participar 6 . Es de señalar que esta dinámica no puede ser supuesta meramente a partir de su régimen de propiedad cooperativa, ya que la importancia de los mecanismos participativos varía en diferentes experiencias cooperativas históricas. Todo ello nos orienta a pensar en una variable cultural. Así, se reconocen en estos mecanismos participativos dispositivos de las convenciones cívicas que determinan los procesos de innovación de productos ${ }^{7}$.

El proceso administrativo y contable del MP se fortaleció con un software de cobro, que supuso cursos de capacitación para los trabajadores de las cajas. EI MP también orientó su planificación contable para adquirir cámaras de frío que le permitan mantener los alimentos frescos. Otro tanto en la incorporación de medios de transporte para apoyar la logística de productores/as.

Para el manejo de la comunicación oficial, el propio proceso participativo decisorio se apoyó nuevamente en las lógicas de la convención industrial. Frente a la pregunta sobre por qué el MP contrata a una profesional en manejo de redes de forma externa, la respuesta general ha sido: la calidad del trabajo realizado, la importancia de su regularidad y la jerarquización de ese tipo de competencias en el contexto actual.

El MP aprovecha sus vínculos con profesionales del Instituto de Producción Popular para confeccionar e implementar sondeos de opinión en el barrio céntrico donde se encuentra emplazado. Con ello se apuntó a evaluar su oferta de productos, con estudios de mercado basados en la composición de la demanda, y apoyar de esta manera los procesos decisorios participativos.

Otro de los casos analizados es un autoservicio (ASC) que comenzó sus actividades en 2002. Esta es una empresa pyme de capital privado familiar compuesta por cuatro integrantes; han sumado 4 empleados, los cuales tienen un sector asignado dentro del comercio y también cuentan con conocimientos suficientes para desempeñar las funciones del resto de los compañeros de trabajo, en caso de precisarse.

\footnotetext{
${ }^{6}$ En entrevista se nos comenta que esta participación contiene en general a un número de productores que acompañan la experiencia desde sus inicios.

${ }^{7}$ Según las herramientas teóricas del enfoque que recuperamos para nuestro análisis cultural de la innovación las convenciones son "referencias para los individuos en sus transacciones" así como "toda forma colectiva que asegura la compatibilidad entre las acciones individuales" (Duvernay, 1998:18). De esta manera la noción de convención opera un rol similar que el precio de mercado del análisis neoclásico.
} 
Puede observarse que esta organización pone especial énfasis en las relaciones entre individuos y en la mejora de los procesos de comunicación interna, dos aspectos indispensables en la gestión del conocimiento tácito (Krieger, 2001). En términos específicos, la experiencia personal de los integrantes del ASC (determinada, a su vez, por factores intangibles como las creencias, valores, puntos de vista, intuiciones, etc.) incide plenamente en la organización del comercio y en la planificación de las actividades, aportando ventajas competitivas que pueden proyectarse a través del tiempo que, a su vez, construyen la identidad de la organización, sin posibilidad de ser imitada por sus competidores.

Sobre las nuevas formas de distribución o comercialización, la organización también acude a la consulta interna y a la visión de los empleados para tomar decisiones. En términos de innovaciones blandas, siempre son consideradas las opiniones 0 sugerencias de los empleados, conversando los temas entre todo el equipo de trabajo, definiendo lo que creen más conveniente para ese momento con la motivación de lograr mayor participación en el mercado, aumentar las ventas y con ellas, las utilidades.

Aquí puede identificarse una de las pautas de la cultura organizacional definida por Krieger (2001), la cual se estructura sobre la base de interacciones particulares que se establecen entre personas dentro de una organización (en este caso, los dueños del ASC y sus empleados), con la finalidad de afrontar simultáneamente una situación problemática y concebir juntas una solución.

En cuanto a los indicadores de cultura organizacional, puede delimitarse un clima organizacional orientado al desarrollo de competencias de sus integrantes y al aprendizaje organizacional, sumado a una cultura cooperativa en relación a las expectativas grupales, dando lugar a una organización que resuelve sus conflictos internos a través de la negociación. Sobre los valores organizacionales, pueden destacarse la importancia que el equipo de trabajo aporta a la ética y a la moral organizacional, priorizando los principios de confianza, reciprocidad y justicia entre todos sus integrantes. En torno a las presunciones básicas, se destaca el compromiso de la organización con los valores, las normas y los objetivos estratégicos, como así también, su predisposición a enfrentar desafíos, aprovechar oportunidades, desarrollar la creatividad y estar abiertos al aprendizaje y al medio. Esta situación se genera bajo el fomento de las relaciones interpersonales, el trabajo en equipo y el cuidado del medio ambiente de trabajo.

La cultura material del ASC está representada por diversos recursos tangibles e intangibles, como así también, por los conocimientos científicos de sus integrantes. Sobre la incorporación de innovaciones duras - por ejemplo, nuevas tecnologías - la 
entrevistada destaca, en primer lugar, el acceso a compras con tarjetas de crédito desde el inicio de sus actividades y, como última incorporación, la trasferencia bancaria de los clientes para el pago de los pedidos entregados en sus domicilios.

En cuanto a la aparición de convenciones que permiten establecer un orden entre las personas, en el Autoservicio San Cayetano puede presentarse la convención doméstica, la cual destaca la calidad humana de los individuos y el sentido de pertenencia por tradición. Si bien esta empresa familiar toma en cuenta las opiniones (y decisiones) de sus clientes para ofrecer un servicio integral acorde a los requerimientos del mercado, y también, prioriza sus recomendaciones al momento de contratar nuevo personal, por otro lado, son las normas de conducta, la buena educación y los lazos de amistad entre los miembros de la organización los dispositivos prioritarios para las normas generales de convivencia y relacionamiento.

Por otro lado, la presencia de clientes y competidores sumada a la comercialización de bienes y prioridad de la vinculación por medio de contratos, son características primordiales de la convención mercantil

En los casos analizados en este trabajo hasta el presente, observamos que las organizaciones tienen en general una proporción de trabajadores pertenecientes a los sectores vulnerables de la sociedad. Las modalidades de trabajo y la disposición a resolver problemáticas emergentes está presente porque poseen condiciones de adaptabilidad, sin embargo son organizaciones más frágiles a turbulencias y eventos no esperados. No cuentan con recursos para realizar adaptaciones tecnológicas importantes, aun cuando se logren implementar ciertas innovaciones apropiadas.

El contexto presente de pandemia ha agravado la crisis de arrastre que tenía la economía argentina. EI MP ha incorporado protocolos sanitarios de circulación interna y productos sanitizantes en su comercialización, como otros mercados del rubro. Sin embargo, la apuesta más interesante radica en su tendencia a apoyarse en los vínculos con su organización madre (CTEP) del mismo modo que se hizo históricamente para la incorporación de personal. La representatividad en el espacio de militancia en el campo de la economía popular es un principio de legitimación que atraviesa a toda la cultura organizacional, un determinante de la racionalidad actoral y un eje de solidaridad sobre el que se refugia la organización para sobrellevar un presente de múltiples riesgos. Sin embargo, y a pesar de la solidez que significa este vínculo, está en juego también la capacidad y posibilidades de la organización central para realizar apoyos permanentes. También es necesario evaluar si la prolongación o la etapa pos-pandemia pueden demandar modificaciones en las prácticas laborales que se puedan incorporar con facilidad. La principal conclusión a la que llega el presente escrito es que esta combinación entre convención cívica e industrial 
determinará gran parte de los procesos de innovación organizacional que le permitan sobrevivir un presente crítico. Dicha combinación se reproduce en la incorporación de tecnologías de diferentes tipos.

En el caso del autoservicio, uno de sus objetivos es la incorporación y permanencia de clientes brindando la mejor atención. Si bien las visitas de los proveedores y la rotación de los productos influyen en el stock del comercio bajo estudio, la preferencia de compra por parte de los clientes de algún producto por sobre otro también incide en la selección de la mercadería por comprar. El ASC tiene, además, permanente contacto con otras organizaciones del mismo rubro o similar porque "sería favorable para el crecimiento de todos los comercios". En consonancia con las disposiciones del nuevo contexto, el autoservicio notó una incidencia negativa de esta medida sobre el desarrollo de su comercio, en relación al poco contacto que pueden mantener con los clientes, provocado notables pérdidas de las ganancias planificadas con anterioridad.

\section{ACTIVIDAD EN LA CRISIS}

Es conocido que varias organizaciones pudieron obtener beneficios e incrementando las ganancias, también sabemos que la mayoría sortea graves dificultades. Si bien los emprendimientos deban enfrentar recurrentemente circunstancias críticas, tal como en la crisis financiera de 2008, la situación actual es más compleja porque afecta la vida misma de los sujetos y la recesión desencadenada es, en su velocidad y escala mundial, diferente a todos los anteriores procesos.

Los datos sobre los cuales se reflexiona están restringidos a un sector, pero se puede inferir que la tendencia es similar, asimismo la información obtenida coincide con otros informes donde se detalla la reducción o suspensión de personal, cierres temporales o definitivos, incertidumbre sobre la oportunidad de reapertura. De hecho, las evaluación cultural se refiere a dos casos, aun cuando las prácticas de gestión que se detallan ha sido observadas en otras organizaciones de la misma rama.

Un inconveniente en todo rubro es la reducción en la capacidad ventas como ocurre a las organizaciones evaluadas, en otros casos puede ser de producción. La pérdida repentina de la demanda afecta la capacidad de funcionamiento y sostenimiento de los compromisos adquiridos.

Respecto al personal, se han presentado circunstancias en las cuales se necesita atender familiares, dedicación al cuidado de los hijos por escuelas cerradas y la movilización se encuentra reducida. Por otra parte se han producido interrupciones en las cadenas de suministros, lo que afecta el servicio a clientes, en otro caso podría significar interrupción de un proceso. Es decir, todos los componentes de incertidumbre, pérdida de confianza en el saber arraigado y las mismas funciones 
aceptadas en las instituciones vinculadas a la producción se han puesto en cuestionamiento. Los postulados de la sociedad de riesgo se encuentran presentes y condicionando a los sujetos.

Las tendencias post-pandemia pueden identificarse en los siguientes aspectos demandados: desintermediación (porque hay mayor demanda de bajo precio y mayor velocidad), digitalización, diversificación, sistematización y accesibilidad.

Las organizaciones consultadas señalaron que se han focalizado en atender tres aspectos, aun cuando fueron realizando experiencias y de modo improvisado, pero fueron la cadena de suministro, los trabajadores, y la relación con los clientes. Han tenido que enfrentarse al dilema de realizar la operación (transacción) y el cumplimiento del control interno, donde la respuesta unánime ha sido garantizar la operación con menor riesgo.

Un objetivo relevante para las dos organizaciones son sus clientes, tal como se describió anteriormente. Para empresas dentro de la rama de actividad que habían implementado anteriormente alguna modalidad virtual fue más sencillo, ya que mediante la misma sostuvieron una dinámica más intensa. Pero la diferencia entre el teletrabajo y el efectivo es muy grande. La reingeniería de procesos que está detrás de este cambio implica riesgos y genera incertidumbre en la práctica. Como contrapartida, la transformación digital en los hogares también está rezagada y exhibe alta heterogeneidad. Por otra parte, aun si se logra la infraestructura necesaria su potencial es limitado: hay que reasignar tareas y una buena parte no puede realizarse virtualmente (Albrieu R. , 2020). Además, analizando conforme a lo desarrollado por Howard Greenville, vemos que el trabajo remoto desafía a la cultura organizacional en tres aspectos fundamentales:

- El individual: porque modifica la forma de socialización y la construcción de identidad y de sentido de pertenencia.

- El grupal: porque transforma la cohesión y la performance.

- El organizacional: porque cambia la productividad y la inclusión.

Al cambiar el trabajo en sí mismo, se pone bajo presión a la coherencia cultural y se cuestiona tanto como se hacen las cosas (prácticas) como los valores que la sustentan, aquello que significan ${ }^{8}$. Si bien la cultura organizacional, por naturaleza y constitución, nunca está bajo el control de la organización completamente, se puede verificar que es susceptible de sufrir orientaciones más precisas de los niveles decisorios en condiciones presenciales.

\footnotetext{
${ }^{8}$ En términos de Bourdieu el habitus y el campus.
} 
En este contexto, a las organizaciones les ha resultado difícil implementar operaciones distintas a las que se realizaban. Para la rama de actividad analizada, la condición de esencial (rama de alimentación) les permitió sostener la actividad a pesar de las restricciones. En uno de los casos se realizaron intentos de realizar una innovación para ventas digitales, sin embargo no se había avanzado satisfactoriamente. Las prácticas internalizadas y pautas adquiridas contrastaban con la relación interpersonal en el servicio al cliente, quienes por otra parte tampoco no exigían este sistema, socialmente las organizaciones son reconocidas por un trato directo. Alguno de los trabajadores describe esta si bien lo interpreta como importante, conlleva una alteración en su identidad laboral, e igualmente no se imaginaban haciéndolo, es decir que retrospectivamente no existe una imagen que justifique la nueva actividad a pesar de ser vital.

Centralmente se realizaron actividades que privilegiaron las operaciones por sobre los tradicionales controles internos, hecho que fue aceptado porque se dirigen resolver una necesidad coyuntural. Estas operaciones no implicaron un cambio cultural ni identitario, y contaban con la aceptación social dada tanto por miembros de la organización como por clientes reconocidos. No era necesario realizar una adaptación general ni dividir funciones nuevamente. Las organizaciones consideran factible introducir modalidades virtuales en las ventas, considerando se han convertido en una necesidad, sin embargo deberán enfrentar pautas culturales antes que tecnológicas. Según Howard-Grenville (Howard Grenville, 2020) para hacer sostenible a la cultura cuando el trabajo es remoto se requiere operar en tres ejes o dimensiones:

A. Hacer la cultura visible: la censura implica invalidación de la cultura, resaltar los hábitos y recordárselos a los miembros de la organización cuando están en acción, actualiza la cultura. Corregir las prácticas que se alejan de los valores vuelve visible por oposición los rasgos culturales que se quieren rescatar. En el caso de las organizaciones analizadas en este trabajo esto no se produjo, incluso la cultura organizacional no es una de las prioridades, por tanto las actitudes fueron en general de rechazo de las modificaciones a las prácticas y representaciones propias

B. Actualizar el repertorio cultural: acomodar nuevas prácticas que son coherentes y refuerzan la cultura organizacional. En los emprendimientos entrevistados este fenómeno no se produjo, tampoco se evaluó la importancia de esta incorporación sino que se pudieron resolver problemas de la coyuntura con elementos y prácticas ya existentes. 
C. Usar la disrupción para reforzar el núcleo cultural: compartir las historias del pasado que permiten ligar con las fortalezas culturales actuales.

\section{CONCLUSIONES}

Si bien las observaciones que presentamos tienen las restricciones de limitarse a una rama de actividad y consistir en entrevistas que involucran pocos casos, se considera que puede ser de utilidad realizar una planteo objetivo sobre algunas organizaciones han gestionado la crisis de la pandemia para sistematizar algunas actividades y dificultades en circunstancias complejas.

En escenarios de crisis generalizada es necesario atender a las amenazas cotidianas, pero resulta central detallar y prestar atención a los nuevos componentes que indiquen riesgo y reconocer a sectores con los cuales es posible sostener estrategias cooperativas conjuntas.

Los casos entrevistados, observaciones en otros sectores y la literatura existente demuestran que los recursos de capital y tecnológicos son importantes, pero las estrategias gerenciales pueden ser óptimas en la reconversión y aprovechamiento de los espacios que permita una crisis devastadora para las Pymes como la que se enfrenta en la actualidad.

En este sentido, la cultura e identidad adquirida en las actividades son factores relevantes siempre en toda organización, y su desconocimiento puede impedir realizar adaptaciones que permitan sobrellevar turbulencias, e incluso el análisis realizado con Duvernay nos permite operar mediante dispositivos que orientan las prioridades. Los actores no pueden, sin una orientación adecuada, realizar actividades para las cuales no encuentran los pilares que conforman su identidad laboral y principalmente si las modificaciones aplicadas o pensadas para aplicar entran en contradicción con pautas culturales.

A partir de esta interpretación vemos que resulta conveniente determinar las modificaciones y cambios que puedan realizarse en procesos y estructuras a fin de reconocer las nuevas posibilidades de adaptarlos a las prácticas culturales preexistentes, observando las nuevas actividades en términos coordinación o desplazamiento de las prácticas y pautas existentes. La gerencia, ante cualquier situación de emergencia puede aprovechar el reconocimiento de la cultura organizacional, factor no siempre atendido ante turbulencias severas y entornos con complejidades. 


\section{REFERENCIAS IBLIOGRÁFICAS:}

Albrieu, R. (2020). Evaluando las oportunidades y los límites del teletrabajo en Argentina en tiempos del COVID 19. Buenos Aires: CIPPEC.

Beck U (2002) La sociedad de riesgo global; Ed. Siglo XXI; Madrid; España

Beck U. (1997) La reinversión de la política: hacia una teoría de la modernización reflexiva, en Beck, Giddens y Lash, "Modernización reflexiva. Política Tradición y estética en el orden social moderno". Ed. Alianza, Madrid.

Caila, M; (2006) Sociedad de riesgo y problemática ambiental' - Revista Ambiental" Editorial Laborde - Noviembre. ISSN 0328-1051

Caila,M y Ulla V. (2016) Política, Sociedad y Naturaleza. El desarrollo sustentable en el capitalismo actual -- Revista Ambiental" - Editorial Laborde - ISSN 03281051

Camou, A., Estudio preliminar, en Camou, A., "Los desafíos de la gobernabilidad", FLACSO-Plaza y Valdés, M México D.F, 2001.

Duvernay, J. (1998); “Economía de las convenciones”; Buenos Aires, Piette.

Harari, Yuval, (2020) "Superaremos la pandemia”; entrevista en XL semanal. Abril

Krieger, M. (2001); "Sociología de las organizaciones"; Buenos Aires, Prentice Hall.

Nonaka y Takeuchi (1995); "The Knowledge creating company".

Terán Mantovani, Emiliano. (2019) "Por qué hablamos de crisis civilizatoria? Breve genealogía de nuestro actual tiempo extraordinario", en Observatorio de Ecología Política de Venezuela.

\section{Artículos en Sitios Web}

- "Preguntas y respuestas sobre la enfermedad por coronavirus (COVID-19)" (2020);

Disponible en Sitio Web Oficial de la Organización Mundial de la Salud (OMS): https://www.who.int/es/emergencies/diseases/novel-coronavirus2019/advice-for-public/q-a-coronaviruses [Fecha de Consulta 10 de Septiembre del 2020].

https://www.cepal.org/es/noticias/alicia-barcena-la-pandemia-covid-19-es-un-llamadorepensar-desarrollo-urbano-avanzar-un

Albrieu, R. (12 de Septiembre de 2020). cippec. Obtenido de https://www.cippec.org/textual/teletrabajo-regulacion-en-argentina/ 
Howard Grenville, J. (2020). How to sustain your organization's culture when everyone is remote. . MIT Sloan Managment Review . 